subsequently every six months. All new pregnancies are followed for exposure and outcome.

Results To date 496 women have signed up, with 63 new pregnancies notified by study members: recruitment is now underway across Canada. The results here are from 385 early recruits (mean age 31.6 years) and we consider only first pregnancies from the baseline questionnaire. We know of 174 first pregnancies (90 welders; 84 electricians): 3 welders chose not to give this information. Elective abortion was frequent, particularly in welders: 64 welders and 66 electricians continued the pregnancy. Spontaneous abortion rates were similar (welders 12/64; electricians 16/66). Only 25 welders and 11 electricians were working in their trade at the start of their first pregnancy and elected not to terminate it. Among these, spontaneous abortion rates were $40 \%(10 / 25)$ for welders and $18 \%(2 / 11)$ for electricians. In a logistic regression allowing for age and cumulative months in the trade at conception, the risk of miscarriage for welders in their trade was raised $(\mathrm{OR}=3.5195 \%$ CI 1.19-10.37). No marked effect was seen on live birth weight (welders $7.25 \mathrm{lbs}$; electricians $7.43 \mathrm{lbs}$ ) but infertility $>12$ months was more likely to be reported by welders: 11/201 welders and 3/184 electricians were working in their trade at the start of a period of infertility $\left(\mathrm{X}^{2}=4.05 \mathrm{p}=0.04\right)$

Conclusions Early data from WHAT-ME are consistent with some reproductive hazard to welders. The cohort's increasing size and prospective data collection will serve to confirm or refute these results.

\section{THE CHANGING SHIFT PATTERN AND OVERTIME AFFECT NURSES' MENSTRUAL CHARACTERISTICS}

W S Chin, Shiao, Guo. National Taiwan University, Taipei, Taiwan

\subsection{6/oemed-2013-101717.218}

Objective The aim of this study was to determine whether the frequency of changing work shift and overtime are associated with registered nurses' menstrual characteristics.

Method Female nursing staff aged 20-45 years and working at hospitals were invited to participate in the study. Menstrual characteristics and work shifts were recorded daily for 180 days. A multivariate logistic model was performed. Important confounders such as age, educational level, occupational category, regular excise habit, and major earner of family were adjusted.

Result A total of 330 nurses and 1,437 menstrual cycles were collected and eligible for the final analysis. The adjusted odds ratio for shorter bleeding time ( $\leqq 3$ days) was associated with changing work shift (involved one night shift) more than 3 times between menstrual cycles were $2.2(95 \% \mathrm{CI}=1.1-4.3)$. The adjusted odds ratios for longer menstrual cycle lengths (>40days) due to changing work shift (involved one night shift) more than 3 times between menstrual cycles were 4.7(95\% CI = 3.1-7.1). The adjusted odds ratios for dysmenorrhea due to overtime more than 40 hours during 28 days before menstrual were 2.9 (95\% CI = 1.6-5.2).

Conclusion The high frequency of changing work shift (esp. from day shift change to night shift or from evening shift to night shift) and overtime (>40 hours/ 28days) may affect childbearing aged female nurses' reproductive function.

\section{WORK RELATED STRESS AND INCREASED RISK OF LOW BIRTH WEIGHT, PREGNANCY-INDUCED HYPERTENSION AND PREECLAMPSIA}

${ }^{1} \mathrm{~T}$ Brand, ${ }^{2}$ Milder. ${ }^{1} \mathrm{AMC} / \mathrm{Coronel}$ Institute, Amsterdam, Nederland, ${ }^{2}$ National Institute for Public Health and the Environment, Bilthoven, Nederland

\subsection{6/oemed-2013-101717.219}

Purpose On request of the National Institute for Public Health and the Environment, the present study was set up to find studies regarding an association between work related stress and pregnancy complications, pregnancy-induced hypertension and preeclampsia and to evaluate the present level of evidence.

Methods PubMed was used to find studies published between 1990 and April 2012, using a search strategy taking into account pregnancy complications, pregnancy-induced hypertension, preeclampsia and work related stress or burnout. For assessment of the quality of the studies, a score was calculated adapted from Nieuwenhuijsen et al.[1].

Results 17 studies were found on preterm birth, low birth weight, spontaneous abortion, pregnancy-induced hypertension and preeclampsia. Using the studies of the highest quality, work related stress was significantly associated with a higher risk of pregnancy-induced hypertension and preeclampsia and also a significant higher risk albeit to a slightly lesser extent, on a lowering of the birth weight of about 150 gram. The association of work related stress and preterm birth or spontaneous abortion was unequivocal.

Conclusions Clear evidence was found that work related stress during pregnancy is strongly associated with pregnancy-induced hypertension and preeclampsia and to a slightly lesser extent with a lowering of the birth weight of about 150 gram. These findings underscore the need for attention and reduction of work related stress during pregnancy in order to prevent the work related effects. Therefore, it was decided to develop an information brochure on work related stress and pregnancy that will be added to the communication toolkit 'Kinderwens, zwangerschap en werk' which can be found on the website http:// toolkits.loketgezondleven.nl/

\section{SHIFT WORK AND SPONTANEOUS ABORTIONS}

${ }^{1}$ B E M Moen, 'Waage, ${ }^{2}$ Ronda, ${ }^{3}$ Magerøy, 'Pallesen, 'Bjorvatn. 'University of Bergen, Bergen, Norway; ${ }^{2}$ University of Alicante, Alicante, Spain; ${ }^{3}$ Haukeland University Hospital, Bergen, Norway

\subsection{6/oemed-2013-101717.220}

Objectives Our aim was to study the relationship between spontaneous abortions among nurses working three different types of shift schedules; permanent day shift, three shift rotation, and permanent night shift.

Methods From a cohort of nurses in Norway established in 2008, we identified 1083 female workers who worked the same type of shift schedule in 2008 and one year later. Information on age, years worked as a nurse, weekly work hours, job demands, job control, smoking, consumption of alcohol, caffeine, spontaneous abortions the past year and throughout life was obtained by questionnaires. The relationship between spontaneous abortion and type of shift schedule was analysed by logistic regression analyses, adjusting for job strain and life-style factors. The analyses were performed in two strata, above and below 30 years of age.

Results An increased risk for experiencing spontaneous abortions the past year was found among nurses who worked only night shifts compared to those who worked only day shifts, 